\title{
Prevalence and Analysis of Drug Resistance Pattern of MDR-TB in Retreatment Cases at Allied Hospital, Faisalabad, Pakistan
}

\author{
Sana Arif ${ }^{1}$, Qamar Mehboob ${ }^{2}$, Waqar Arif ${ }^{3}$
}

1 House Officer, Allied Hospital, Faisalabad Pakistan

1 Data Collection

2 Associate Professor, Physiology, Faisalabad Medical University, Faisalabad Pakistan

2 Results Compilation, Statistical Analysis

Post Graduate Resident, Plastic Surgery Department, Allied Burn \& Reconstructive Surgery Center Faisalabad

3 Medical University, Faisalabad Pakistan

Data Collection, Manuscript Writing

\author{
Submitted for Publication: 05-01-202 \\ Accepted for Publication 28-09-2020 \\ CORRESPONDING AUTHOR \\ Dr. Qamar Mehboob \\ Associate Professor, Department of Physiology \\ Faisalabad Medical University, Faisalabad Pakistan \\ Email: qamarmehboob89@yahoo.com
}

\section{ABSTRACT}

Background: The prevalence of Multi-Drug Resistance-Tuberculosis (MDR-TB) among retreatment cases is very high. It has become a serious public health issue in Pakistan and can affect greatly TB control strategies. So, we conducted the present research work to update a systematic review of the literature available on MDR-TB cases. Objective: To analyze the resistance pattern of multidrug-resistant tuberculosis. To study the prevalence of MDR-TB among new cases, retreatment cases. Study Design: Cross-sectional study. Settings: Pathology department, Allied Hospital, Faisalabad Medical University, Faisalabad, Pakistan. Duration: Six months from January 01, 2020 to June 30, 2020. Methodology: In this study, we included new and retreatment tuberculosis cases. Gene mutation RpoB, DNA band sequence detected by using machine, Cepheid gene Xpert IV - 4 Modules, Sr \# 806133. Data was collected and analyzed statistically by using SPSS v 20. Results: 858 cases were included of age 12 years $-100 y$ ys with the mean age (in years) $42.139 \pm 16.823$. Two groups of males and females were studied separately. Group I included male patients $\{456(56.64 \%)\}$ and further subdivided into IA [age above 30 years, $\{354(72.84 \%)\}]$ and IB [age below 30 years $\{132(27.15 \%)\}]$. Group II included female patients $\{372(43.36 \%)\}$ and further subdivided into IIA [age above 30 years $\{239(64.25 \%)\}$ ] and IIB [age below 30 years $\{133(35.75 \%)\}$ ]. Out of 858 cases, $347(40.44 \%)$ were New cases, 13 (1.51\%) New cases with HIV and 408 (47.55\%) were Previously Treated patients (PT). 30 $(3.45 \%)$ cases were unknown (UK). 28 (3.26\%) cases were brought by different lady health workers (LHW) and relapse cases were $32(3.73 \%)$. Overall statistical analysis was found to be highly significant ( $p=0.000, d f=1, C I=95 \%)$. According to our results, in $517(60.26 \%)$ patients, Mycobacterium Tuberculosis was not detected (MTB-ND). In 214 (24.94\%) cases, Mycobacterium Tuberculosis was detected (MTB-D), out of which 13 (6.07\%) cases were of First-Line Multi Drug Resistance (MDR). Age of MDR cases was in between 17- 70 years with the mean age (in years) $37.307 \pm 17.637$. Our results showed MDR more in males (53. 85\%) as compared to female patients (46.15\%). Our results showed a significant correlation between status of all cases and their reports $(p=-.050)$. For MTB-ND cases $p=0.000, d f=1, C I=95 \%$ and for invalid cases $p=$ $0.000, \mathrm{df}=5, \mathrm{CI}=95 \%$. Conclusion: There is a great need to implement national tuberculosis guidelines fully. In order to prevent further emergence of drug resistance, we should focus on effective implementation of directly observed therapy short courses.

Keywords: Multi drug resistance, Retreatment cases, Tuberculosis.

How to Cite: Arif S, Mehboob Q, Arif W. Prevalence and Analysis of Drug Resistance Pattern of MDR-TB in Retreatment Cases at Allied Hospital, Faisalabad, Pakistan. APMC 2020;14(4):331-5. DOI: 10.29054/ APMC/2020.994

\section{INTRODUCTION}

Tuberculosis (TB) has remained a global health problem for last many years. ${ }^{1}$ In present condition, there are 22 countries that have almost $80 \%$ of the world's TB cases. Unfortunately, documented data of these high burden countries do not reflect the actual figures because of improper data collection processes, or incomplete coverage. ${ }^{2}$ In 2020, Gauri Suhas Kulkarni reported that in most of the countries MDR-TB is a major health problem which can be an obstacle to effective TB control. ${ }^{3}$ Multiple studies have been conducted for the assessment of the risk factors leading to the increase ratio of MDR-TB. ${ }^{4}$ Multidrug-resistant tuberculosis (MDR-TB), resistant to both isoniazid and rifampicin, is a serious health problem, not only for the public health sector but also to the general physicians, as well as the pulmonologists of Pakistan, who are treating TB. ${ }^{5}$

According to the latest Global TB Report, during 2018, approximately 484,000 people developed rifampicinresistant (RR) /MDR-TB globally, 3.4\% new and $18 \%$ of previously treated cases of TB, were rifampicin resistant (RR) /MDR-TB. 6 Only in China, $24 \%$ of previously treated cases and $7.1 \%$ of new cases had MDR-TB, more than the global averages. ${ }^{7}$ So, strength MDR-TB management is very difficult to control the prevalence of MDR-TB. ${ }^{8}$ 
Due to poverty and some other risk factors, the geographic distribution of the disease may vary worldwide, as well as within the country.9,10 Delayed drug susceptibility testing before MDR-TB diagnosis could result in further transmission of drug resistant $\mathrm{TB}$ strains and use of inappropriate regimen of second line drugs would increase the risk of their resistance. ${ }^{11}$ In 2019, Gelaw YA reported that low altitude areas and high temperature increases the TB incidence. ${ }^{12,13}$

Pakistan ranks 5th amongst the high burden countries in the world. The prevalence, incidence and mortality per 100,000 populations per year from TB in Pakistan are 340, 265 and 27 respectively. ${ }^{14}$ According to a report of the Global Tuberculosis Community Advisory Board, in Pakistan, the number of TB patients is increasing rapidly at an estimated rate of 27,000 new cases per year..$^{15}$ World Health Organization (WHO) reported that during 2018, 562000 TB cases fell ill in Pakistan out of which 44000 cases died. While 28000 people fell ill with drug-resistant $\mathrm{TB}^{16}$. So, the current study was planned to determine the prevalence and drug resistance patterns of MDR-TB in retreatment case at FMU, Allied Hospital, Faisalabad.

Objective: Our study aims to study the prevalence of MDR-TB among new cases and retreatment cases and to analyze the resistance pattern of MDR-TB. To find out the factors associated with RR-TB among DR-TB patients and also to determine the correlation of RR-TB with MDR-TB.

\section{METHODOLOGY}

Study Design: Cross-sectional study.

Settings: Pathology department, Allied Hospital, Faisalabad Medical University, Faisalabad, Pakistan.

Duration: Six months from January 01, 2020 to June 30, 2020.

Sample Technique: Non-probability consecutive sampling.

Sample Size: 858 cases

Inclusion Criteria: Those who were taking anti TB treatment. Previously treated, relapse and new cases. Male, female

Exclusion Criteria: Extra pulmonary TB. Patients with no anti-TB drug treatment.

Data Collection Procedure: This cross-sectional multisite study was carried out in six months from January 01, 2020 to June 30, 2020 at Allied Hospital Faisalabad, and included 858 cases. Gene mutation RpoB, DNA band sequence detected by using machine, Cepheid Gene Xpert IV - 4 Modules, Sr \# 806133, placed at Bio Safety Lab II (BSL-II). Data was collected and analyzed statistically.

\section{RESULTS}

858 cases were included of age 12 years - 100yrs with the mean age (in years) $42.139 \pm 16.823$. Two groups of males and females were studied separately. Group I included male patients $\{456(56.64 \%)\}$ and further subdivided into
IA [age above 30 years, $\{354(72.84 \%)\}]$ and IB [age below 30 years $\{132(27.15 \%)\}]$. Group II included female patients $\{372(43.36 \%)\}$ and further subdivided into IIA [age above 30 years $\{239(64.25 \%)\}$ ] and IIB [age below 30 years $\{133(35.75 \%)\}]$. (Table: 1, 2, Fig: 1). Statistical analysis showed $\mathrm{p}=0.000, \mathrm{df}=1, \mathrm{CI}=95 \%$.

Table 1: Age (in years). $\mathrm{n}=858$.

\begin{tabular}{|c|c|c|}
\hline \multicolumn{3}{|c|}{ Statistics } \\
\hline \multicolumn{3}{|c|}{ Age } \\
\hline \multirow{2}{*}{$\mathbf{N}$} & Valid & 858 \\
\hline & Missing & 1 \\
\hline \multicolumn{2}{|c|}{ Mean } & 42.1399 \\
\hline \multicolumn{2}{|c|}{ Std. Deviation } & 16.82324 \\
\hline \multicolumn{2}{|c|}{ Minimum } & 12.00 \\
\hline \multicolumn{2}{|c|}{ Maximum } & 100.00 \\
\hline
\end{tabular}

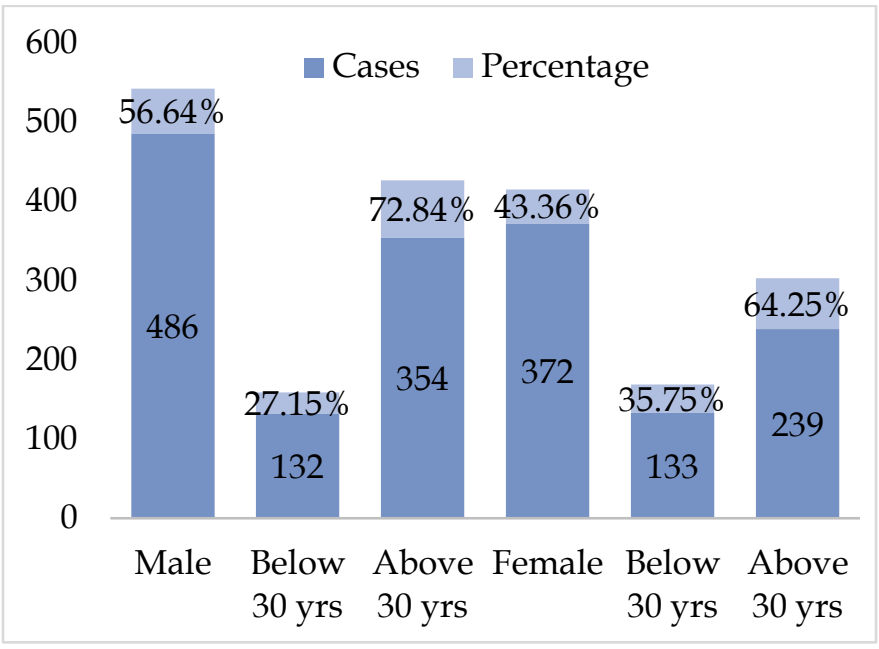

Figure 1: Age and gender distribution of study subjects $(n=858)$

In our study, we included, out of 858 cases, 347 (40.44\%) New cases, 13 (1.51\%) New cases with HIV, 408 (47.55\%) Previously Treated patients (PT). 30 (3.45\%) cases were unknown (UK). 28 (3.26\%) cases were brought by different lady health workers (LHW) and relapse cases were $32(3.73 \%)$. (Table 2$)$

Table 2: Details of status of the cases $(n=858)$

\begin{tabular}{|c|c|c|}
\hline Status & Cases & Percentage \\
\hline New & 347 & 40.44 \\
\hline New. HIV & 13 & 1.51 \\
\hline PT & 408 & 47.55 \\
\hline UK & 30 & 3.45 \\
\hline LHW & 28 & 3.26 \\
\hline F.up New/PT (Relapse) & 32 & 3.73 \\
\hline
\end{tabular}

PT = Previously Treated, UK= Unknown, LHW= Cases brought by Lady Health Worker, F.up= Follow up/Relapse 
In our study, we used following definitions: ${ }^{17}$

$>$ New case: All TB cases that never took treatment for $\mathrm{TB}$ or has taken the treatment for less than 1 month.

$>$ Retreatment case: Includes cases of failure, relapse, treatment after default, and others.

$>$ MDR-TB case: A suspected case who is bacteriologically confirmed of Tuberculosis and has Mycobacterium TB resistant to first-line anti-TB drugs, either with or without resistance to other antiTB drugs, based on drug sensitivity testing (DST) results.

According to our results, in $517(60.26 \%)$ patients, Mycobacterium Tuberculosis was not detected (MTBND). In 214 (24.94\%) cases, Mycobacterium Tuberculosis was detected (MTB-D), out of which $13(6.07 \%)$ cases were of First-Line Multi Drug Resistance (MDR). These cases included 3 (23.08) new cases and 10 (76.92\%) were Previously Treated patients. Out of 13 MDR cases, 7 (53. $85 \%)$ were male and $6(46.15 \%)$ were female. Age of MDR cases was in between 17- 70 years with the mean age (in years) $37.307 \pm 17.637$.

Our results showed MDR more in males (53. 85\%) as compared to female patients (46.15\%). We did not check $2^{\text {nd }}$ line MDR because of limitations of Gene Xpert. Line Prob Assay (LPA) was not functioning. In 201 (93.93\%) cases, Mycobacterium Tuberculosis was detected but they did not show MDR so we labeled them as NonResistant/ not detected, cases (ND). Errors appeared in $117(13.64 \%)$ cases due to different factors like temperature or in correct sequence of nucleotide bases. Reports of 10 (1.16\%) cases were invalid. (Table 3,4)
Table 3: Categories of multidrug-resistant tuberculosis cases $(\mathrm{n}=858)$

\begin{tabular}{|l|l|l|l|l|}
\hline \multicolumn{2}{|l|}{ Status } & \multicolumn{2}{l|}{ Cases } & Percentage \\
\hline MTB-ND & & 517 & 60.26 \\
\hline MTB-D & & 214 & 24.94 \\
\hline & MDR & & 13 & 6.07 \\
\hline & & New & 3 & 23.08 \\
\hline & & PT & 10 & 76.92 \\
\hline & & Male & 7 & 53.85 \\
\hline & & Female & 6 & 46.15 \\
\hline & ND & & 201 & 93.93 \\
\hline Errors & & & 117 & 13.64 \\
\hline Invalid & \multicolumn{2}{|l}{} & 10 & 1.16 \\
\hline
\end{tabular}

MTB-ND $=$ Mycobacterium Tuberculosis Not Detected, MTB-D $=$ Mycobacterium Tuberculosis Detected, $\mathrm{MDR}=$ Mycobacterium Tuberculosis Detected with Multi Drug Resistance, ND = Mycobacterium Tuberculosis Detected with No Resistance

Table 4: Age of MDR cases $(n=13)$

\begin{tabular}{|l|l|l|}
\hline \multicolumn{2}{|l|}{ Statistics } \\
\hline \multicolumn{2}{|l|}{ MDR } & 13 \\
\hline \multirow{2}{*}{ N } & Valid & 846 \\
\cline { 2 - 3 } & Missing & 37.3077 \\
\hline Mean & 17.63701 \\
\hline Std. Deviation & 17.00 \\
\hline Minimum & 70.00 \\
\hline Maximum
\end{tabular}

All the data was collected and statistically analyzed through SPSS v 20. Chi-Square and T- Test were applied, where needed. Our results showed a significant correlation between status of all cases and their reports $(p=-.050)$. For MTB-ND cases $p=0.000, d f=1, C I=95 \%$ and for invalid cases $\mathrm{p}=0.000, \mathrm{df}=5, \mathrm{CI}=95 \%$.

Table 6: Gender status cross tabulation

\begin{tabular}{|c|c|c|c|c|c|c|c|c|c|}
\hline \multicolumn{10}{|c|}{ Gender * Status * Report Cross tabulation } \\
\hline \multicolumn{10}{|c|}{ Count } \\
\hline \multirow{2}{*}{\multicolumn{3}{|c|}{ Report }} & \multicolumn{6}{|c|}{ Status } & \multirow{2}{*}{ Total } \\
\hline & & & New & New HIV & PT & UK & LHW & Relapse & \\
\hline \multirow{3}{*}{ MTB-ND } & \multirow{2}{*}{ Gender } & Male & 78 & & 408 & & & & 486 \\
\hline & & Female & 31 & & 0 & & & & 31 \\
\hline & \multicolumn{2}{|c|}{ Total } & 109 & & 408 & & & & 517 \\
\hline \multirow{2}{*}{ MDR } & Gender & Female & & 3 & & & 10 & & 13 \\
\hline & \multicolumn{2}{|c|}{ Total } & & 3 & & & 10 & & 13 \\
\hline \multirow{2}{*}{ ND } & Gender & Female & 201 & & & & & & 201 \\
\hline & \multicolumn{2}{|c|}{ Total } & 201 & & & & & & 201 \\
\hline \multirow{2}{*}{ Error } & Gender & Female & 37 & & & 30 & 18 & 32 & 117 \\
\hline & \multicolumn{2}{|c|}{ Total } & 37 & & & 30 & 18 & 32 & 117 \\
\hline \multirow{2}{*}{ Invalid } & Gender & Female & & 10 & & & & & 10 \\
\hline & \multicolumn{2}{|c|}{ Total } & & 10 & & & & & 10 \\
\hline \multirow{3}{*}{ Total } & \multirow{2}{*}{ Gender } & Male & 78 & 0 & 408 & 0 & 0 & 0 & 486 \\
\hline & & Female & 269 & 13 & 0 & 30 & 28 & 32 & 372 \\
\hline & \multicolumn{2}{|c|}{ Total } & 347 & 13 & 408 & 30 & 28 & 32 & 858 \\
\hline
\end{tabular}




\section{DISCUSSION}

Multi Drug Resistance-TB is a manmade phenomenon. Factors like poor adherence to treatment, use of inadequate regimens and inappropriate directly observed treatment short-course (DOTS) leads to increase in the drug resistance levels in our country. The improperly treated or undiagnosed patients with resistant strains of TB are being a continuous source of ongoing transmission of resistant strains. In a resourcelimited country such as Pakistan, there are limitations to the affordability and availability of drug sensitivity testing (DST) facilities. So, most of the MDR is diagnosed presumptively based on the lack of response to treatment or relapse of symptoms in TB cases. In present study, our results showed very low prevalence of MDR-TB, namely $0.349 \%$ in new TB cases and $1.165 \%$ in retreatment cases, as compared to other studies.

In present study, our results showed very low prevalence of MDR-TB, namely $0.349 \%$ in new TB cases, $1.165 \%$ in retreatment cases and in new/ re-treatment (both) cases is $1.515 \%$ as compared to other studies. In their study, Rashid A. Khan ${ }^{4}$ et al showed prevalence of MDR-TB in new/ re-treatment (both) cases as $40.2 \%$, Abdul Majeed Akhtar et al ${ }^{18}$ showed $41 \%$ cases, Angrup ${ }^{19}$ et al as 5-12.7\% cases and Datta ${ }^{20}$ et al as $5.75 \%$ cases. Gauri Suhas Kulkar ${ }^{3}$ et al showed $0.10 \%$ in new $\mathrm{TB}$ cases and $4.3 \%$ in retreatment cases while Mistry ${ }^{21}$ et al showed $24-30 \%$ in new $\mathrm{TB}$ cases and $11.67 \%$ in retreatment cases. In another study conducted by Zhengwei $\mathrm{Liu}^{8}$ et al $1.1 \%$ new TB cases and 3.4\% retreatment cases were reported.

In present study we also found less prevalence in new cases $(0.35 \%)$ while high prevalence in retreatment cases $(1.16 \%)$.

After a non-systematic search of the literature, G B Migliori22 et al reviewed the core published documents and guidelines to provide public health workers and clinicians with an updated and easy-to-consult document arising from consensus of Global Tuberculosis Network (GTN) experts., including the recently published MDRTB WHO rapid advice and guidelines. They reported that early diagnosis and successful treatment of MDR-/XDRTB depend on universal DST. In another study forwarded by Angelo Iacobino ${ }^{23}$ et al, it was mentioned that human errors may contribute to the development of DR because of the improper use of anti-TB drugs.

In 2020, Xu-Bin Zheng ${ }^{24}$ et al reported that in China, most of the patients did not receive proper MDR-TB treatment so their delayed diagnosis resulted in poor quality of MDR-TB care. It is the need of the day that regulated patient management, including rapid diagnosis and highquality MDR-TB treatment should be enhanced.

Regarding the limitation of the study, all new and retreatment cases were not subjected to DST because of the limitation of resources. Only suspected patients i.e. category-I failure and category-II failure were subjected to DST. This could be the most probable reason for the low prevalence of MDR-TB in our study.

\section{CONCLUSION}

In Pakistan MDR-TB is a serious public health problem which greatly affects TB control strategies. The prevalence of MDR-TB among retreatment cases is high. The National TB guidelines should be fully utilized by focusing on expansion and effective implementation of directly observed therapy short courses. It will prevent further emergence of drug resistance.

\section{LIMITATIONS}

The study was limited to cases of Faisalabad Division. Nationwide sample collection can change the outcome.

\section{SUGGESTIONS / RECOMMENDATIONS}

We suggest that more and more TB preventive programs should be introduced. The findings of our research work support the recommendations of finding the high rate of MDR and to implement a strategy for those cases that have dropped out of their treatment protocol to control the multi-drug resistance-TB.

\section{CONFLICT OF INTEREST / DISCLOSURE}

No conflict of interest to be declared.

\section{ACKNOWLEDGEMENTS}

We are highly thankful to Associate Prof. Dr. Khalid Hashmi, HOD, Pathology Department, Faisalabad Medical University, Faisalabad, Pakistan and Mr. Mujahid, Incharge BSL II, Pathology Department, Faisalabad Medical University, Faisalabad, for their cooperation and guideline to conduct this research project.

\section{REFERENCES}

1. World Health Organization: global tuberculosis report 2015. [Apr; 2019]

2. World Health Organization. Geneva, Switzerland: World Health Organization; 2011. World Health Organization: tuberculosis prevalence surveys: a handbook. [Apr; 2019].

3. Kulkarni GS, Palwe SD, Patil NP, Telkhade AJ, Kadukar J. Prevalence of multidrug-resistant tuberculosis at a regional drugresistant tuberculosis center of Maharashtra. Indian J Respir Care. 2020;9(1):30-4.

4. Rashid A Khan, Anwar A Shaikh, and Ghulam Q Bulaadi; Incidence of Multidrug-resistant Tuberculosis in Sindh, Pakistan. Cureus. 2019;11(4):e4571.

5. World Health Organization: global tuberculosis report 2013. [Apr;2019]

6. Shibabaw A, Gelaw B, Gebreyes W, Robinson R, Wang S-H, Tessema B. The burden of pre-extensively and extensively drugresistant tuberculosis among MDR-TB patients in the Amhara region, Ethiopia. Plos One. 2020;15(2):e0229040.

7. World Health Organization, Global Tuberculosis Report 2019, World Health Organization, Geneva, Switerzland, 2019.

8. Zhengwei Liu, Mingwu Zhang, Jianmei Wang, Songhua Chen, Beibei $\mathrm{Wu}$, Lin Zhou, et al. Longitudinal Analysis of Prevalence and Risk Factors of Rifampicin-Resistant Tuberculosis in Zhejiang, China. BioMed Research Int. 2020;3159482.

9. Glaziou P, Sismanidis C, Floyd K, Raviglione M. Global epidemiology of tuberculosis. Cold Spring Harb Perspect Med. 2014;5(2):a017798.

10. Touray K, Adetifa IM, Jallow A, Rigby Jan, Jeffries D, Cheung YB, et al. Spatial analysis of tuberculosis in an urban west African 
setting: is there evidence of clustering? Trop Med Int Health. 2010;15(6):664-72.

11. Chen $Y$, Yuan Z, Shen X, Wu J, Wu Z, Xu B. Resistance to SecondLine Antituberculosis Drugs and Delay in Drug Susceptibility Testing among Multidrug-Resistant Tuberculosis Patients in Shanghai. BioMed Res Int. 2016;2016.e2628913.

12. Gelaw YA, Yu W, Magalhães RJS, Assefa Y, Williams G. Effect of Temperature and Altitude Difference on Tuberculosis Notification: A Systematic Review. J Glob Infect Dis. 2019;11(2):63-8.

13. Li X, Wang L, Zhang J, Liu YX, Zhang H, Jiang SW, et al. Exploration of ecological factors related to the spatial heterogeneity of tuberculosis prevalence in P. R. China Global Health Action. 2014;7(1):23620.

14. National TB control program- Pakistan; 2020.

15. Faizan Hashmi; TB cases on the rise in Pakistan Global Tuberculosis Community Advisory Board, April 21, 2019, UrduPoint

16. National TB control program- Pakistan; Profile of Pakistan, WHO Global TB Report 2019 [2020].

17. Central TB Division, Directorate General of Health Services, Ministry of Health and Family Welfare, Nirman Bhavan. Guidelines on Programmatic Management of Drug Resistant TB in India. New Delhi: Central TB Division, Directorate General of Health Services, Ministry of Health and Family Welfare, Nirman Bhavan; 2012.
18. Akhtar AM, Arif MA, Kanwal S, Majeed S. Prevalence and drug resistance pattern of MDR TB in retreatment cases of Punjab, Pakistan. J Pak Med Assoc. 2016;66(8):989-93.

19. Angrup A, Varma-Basil M, Kumar S, Pathak RK, Sharma H, Banavaliker JN, Bose M. Drug resistance among Mycobacterium tuberculosis isolates from private clinics and a dots center in Delhi, India. Southeast Asian J Trop Med Public Health. 2011;42(1):122-7.

20. Datta BS, Hassan G, Kadri SM, Qureshi W, Kamili MA, Singh H et al. Multidrug-resistant and extensively drug resistant tuberculosis in Kashmir, India. J Infect Dev Ctries. 2009;4:19-23.

21. Mistry N, Tolani M, Osrin D. Drug-resistant tuberculosis in Mumbai, India: An Agenda for operations research. Oper Res Health Care. 2012;1(2):45-53.

22. Migliori GB, Tiberi S, Zumla A, Petersen E, Chakaya JM, Wejse C, et al. MDR/XDR-TB management of patients and contacts: Challenges facing the new decade. The 2020 clinical update by the Global Tuberculosis Network. Int J Infect Dis. 2020;92:15-25.

23. Angelo Iacobino, Lanfranco Fattorini, Federico Giannoni, Istituto Superiore di Sanita, Drug-Resistant Tuberculosis 2020: Where We Stand. Appl Sci. 2020;10(6):2153.

24. Xu-Bin Zheng, Vinod K. Diwan, Qi Zhao1, Yi Hu, Judith Bruchfeld, Wei-Li Jiang, et al. Treatment quality and outcome for multidrugresistant tuberculosis patients in four regions of China: a cohort study, Infectious Diseases of Poverty. Open Access BMC. 2020;9:97. 